\title{
Darwinus anarchistus explodens
}

Science et légende de la lutte pour la vie (Louise Michel)

Darwinus anarchistus explodens: Science and the Legend of the Struggle for Life

(Louise Michel)

\section{Claude Rétat}

\section{(2) OpenEdition}

\section{Journals}

Édition électronique

URL : http://journals.openedition.org/aes/2272

DOI : 10.4000/aes.2272

ISSN : 2258-093X

\section{Éditeur}

Laboratoire LISAA

\section{Référence électronique}

Claude Rétat, « Darwinus anarchistus explodens », Arts et Savoirs [En ligne], 12 | 2019, mis en ligne le

25 février 2020, consulté le 02 mars 2020. URL : http://journals.openedition.org/aes/2272 ; DOI :

$10.4000 /$ aes. 2272

Ce document a été généré automatiquement le 2 mars 2020.

Centre de recherche LISAA (Littératures SAvoirs et Arts) 


\title{
Darwinus anarchistus explodens
}

\author{
Science et légende de la lutte pour la vie (Louise Michel) \\ Darwinus anarchistus explodens: Science and the Legend of the Struggle for Life \\ (Louise Michel)
}

Claude Rétat

1 À l'origine de cette contribution, la référence darwinienne chez Louise Michel, prégnante dans l'ensemble de l'œuvre, aussi bien dans l'œuvre d'imagination que dans le vaste corpus des textes encyclopédiques, resté largement manuscrit ${ }^{1}$. Il ne s'agira pas ici de valider ou d'invalider tel ou tel énoncé scientifique, mais d'observer les modalités selon lesquelles un topos s'agence à un imaginaire. En cette fin de siècle, évoquer Darwin, prendre position sur la question de la «lutte pour la vie $»^{2}$, cela n'a rien d'original, et entre dans un jeu idéologique largement codifié. Ainsi, dans le cas de Mirbeau et de son roman Le Jardin des supplices (1899), Louise Lyle a pu montrer comment s'articulaient les allusions à Darwin, la dénonciation du " darwinisme social », un anti-républicanisme anarchiste et une dénonciation des élites politiquesscientifiques ${ }^{3}$.

2 Louise Michel a mis Darwin à son programme de lectures. À l'automne 1885, elle demande à Paul Lafargue qui lui rend visite en prison : «N'oubliez pas de m'apporter [...] le Descent of Man de Darwin, sa lecture fortifiera mon anglais ${ }^{4}$. Elle cite en effet l'ouvrage dans ses manuscrits encyclopédiques, aux chapitres sur la linguistique : « Ce même cébus du Paraguay dit Darwin "fait entendre des sons distincts qui provoquent chez les autres singes des émotions semblables". "

3 Elle met aussi Darwin au programme des lectures d'un personnage de ses romans. Les livres qu'elle injecte ainsi en fiction ne sont jamais insignifiants : ce sont par exemple les Paroles d'un révolté, de Kropotkine (qui apparaissent dans Le Claque-dents, 1889-1890), Le Biribi de Darien (qui apparaît dans La Chasse aux loups, 1891). Dans Les Microbes humains, roman publié en 1886, la lecture de Darwin échoit à la jeune Harriette :

4 L'enfant était entourée de tout ce qui pouvait développer son intelligence (ainsi le seront un jour tous les petits de la race humaine). Elle s'était endormie en lisant. Le 
livre était tombé à terre, un livre grave, on les aime avec passion à cet âge ; c'était Darwin. ${ }^{6}$

5 L'hommage peut sembler équivoque : passion, ou effet somnifère? Mais surtout, il prend place à la fin du roman, peu avant l'assassinat de cette même Harriette : la fillette est la proie d'un « loup » qui s'est introduit dans la « bergerie ».

6 Sous la forme du livre tombé à terre, Darwin vaut ainsi comme signe du présent bestial, sanglant, mais aussi comme signe d'un avenir qui promet le plein développement de "tous les petits de la race humaine" (un jour, comme Harriette qui est une enfant bourgeoise, tous seront enveloppés de culture). Le livre de Darwin prend place à l'articulation de la violence, de la science et de la pédagogie, de l'optimisme du monde futur : la romancière, institutrice et pédagogue en grand (le projet encyclopédique en témoigne), rêve l'éducation de l'humanité. Le jugement sur Darwin, éducateur de la jeunesse et développeur d'intelligence, est donc très positif au moment même où le topos qui l'associe à la violence, à la licence meurtrière au profit du plus fort, est validé. S'il est perçu comme décrivant l'essence du présent (une jungle sanglante), Darwin figure aussi comme conscience scientifique de cet état de fait, donc comme progrès de la conscience, donc comme vecteur de l'évolution humaine.

\section{Dixit Darwin}

7 Les citations de Darwin, à proprement parler, restent peu nombreuses dans l'œuvre encyclopédique : il ne faut pas chercher de discussion serrée, en revanche les allusions sont fréquentes (autour de mots déclencheurs), et il plane, comme autorité tutélaire, sur l'ensemble de l'entreprise. Tout n'est pas resté manuscrit : Louise Michel a publié deux minces opuscules, en 1888 (Lectures encyclopédiques par cycles attractifs), et vers 1894 (Notions encyclopédiques par ordre attractif). Leur minceur témoigne paradoxalement de leur importance aux yeux de l'écrivaine, dont l'objectif était de délivrer une quintessence du savoir, la plus substantielle possible sous le plus petit volume, à l'instar des « résines de l'Inde » dont un « fragment gros comme une tête d'épingle » nourrit longtemps ${ }^{7}$.

8 La première brochure affiche un résumé de Darwin (assez long relativement à l'ensemble, il occupe presqu'une page entière sur seize) :

Tous les êtres organisés sont soumis, dit Darwin, à un certain nombre d'influences.

Tous sont en lutte contre tous, pour conquérir le droit de vivre, c'est la lutte pour

l'existence. ${ }^{8}$

9 Vient à la suite une série d'énoncés: la variabilité des espèces, la transmission héréditaire auxiliaire de la variabilité, la sélection naturelle, l'extinction des individus présentant des "particularités nuisibles", la transmission des particularités qui " assurent un avantage dans la lutte pour l'existence ». En référant en note à " $\mathrm{Ch}$. Darwin, de l'origine des espèces ou des lois de transformation des êtres organisés ${ }^{9}$, Louise Michel fait sonner un mot pour elle fondamental : «transformation $»^{10}$.

10 La dernière page de la brochure oriente Darwin dans un sens développé par plusieurs anarchistes, en réponse aux doctrines du darwinisme social. Pour Louise Michel c'est la lutte pour la vie qui déclenche le groupement social, c'est-à-dire la lutte contre les dangers, contre la violence, contre les oppressions : 
Les hasards de la lutte pour vivre, le combat incessant pour l'existence avaient forcé l'homme à appeler à son aide d'autres hommes. Les premières relations amenèrent des échanges de paroles, de signes, de produits. ${ }^{11}$ citation d'Élisée Reclus :

L'harmonie est la loi de l'univers - C'est dans leur ordre harmonique que sont groupées ces lectures encyclopédiques.

Les dernières pages d'Élysée [sic] Reclus leur serviront d'introduction.

L'homme dit-il a ses lois comme la terre. Vue de haut et de loin, la diversité des traits qui s'entremêlent à la surface du globe [...] présente une image qui n'est pas le chaos, mais un ensemble merveilleux de rythme et de beauté. L'homme qui contemple et scrute cet univers assiste à l'œuvre immense de la création incessante qui commence toujours et ne finit jamais, et participant lui-même par l'ampleur de la compréhension à l'éternité des choses, il peut arriver comme Newton, comme Darwin, à les résumer d'un mot. ${ }^{12}$

Les lignes citées par Louise Michel ont été écrites par Reclus en conclusion de sa Géographie universelle, à laquelle il vient de mettre la dernière main : elles sont publiées par L'Intransigeant du 3 janvier 1894, dans un contexte mouvementé (le cours de Reclus à l'Université libre de Bruxelles a été annulé, il est arrêté, on perquisitionne son domicile). Il explique avoir cherché à exercer un regard unificateur sur la "création incessante ", à travers la diversité des migrations et des peuplements: tous ces mouvements humains, il veut les prendre non tels qu'ils paraissent « au premier abord [...] des faits juxtaposés dans le temps » mais en tant qu'ordonnés par un «rythme », pourvus «d'un sens général exprimable par une loi ${ }^{13}$.

13 Le duo Newton-Darwin, devient, du point de vue de Louise Michel qui cite Reclus, un trio Newton-Darwin-Reclus, et en somme, pour le xix siècle, un duo Darwin-Reclus, dont l'enjeu, brûlant, est de comprendre l'humain et d'en extraire des «lois »: deux voyants, en somme, de la science de l'humanité. La conviction de Reclus, «l'humanité se fait une », constitue aussi son objet scientifique: "Que nos origines aient été multiples ou non, cette unité grandit, elle devient une réalité vivante. » L'entreprise de vastes dimensions (la Géographie universelle) est ainsi tendue (comme chez Louise Michel) par le projet d'un petit livre qui livrerait l'idée organisatrice, le point de l'unité :

Du million de faits que j'ai dû énumérer de chapitre en chapitre, je voudrais extraire une idée générale et justifier en un court volume, écrit à loisir, la longue série de livres sans conclusion apparente que je viens de terminer. ${ }^{14}$

14 Rien d'étonnant donc si Darwin est plutôt appelé à être lui-même quintessencié sous la forme de quelques bribes ou slogans : ainsi se dit et se diffuse une « loi » de l'humanité, dont la brièveté même garantit la haute valeur théorique et permet de toucher l'unité ultime.

\section{Contexte. Le plus fort dans la lutte / Le meilleur dans la joute}

15 Élisée Reclus constitue pour Louise Michel un modèle scientifique revendiqué. Il ouvre et prouve une voie anarchiste du savoir. L'ambition scientifique, la passion du savoir, la conviction que savoir fait partie intégrante de l'épanouissement de l'individu, les réunissent tous deux. Le Darwin anarchiste élaboré par Louise Michel diffère ainsi 
largement de celui qu'a analysé Louise Lyle chez Mirbeau : ici, le scientifique ne sombre pas avec la dénonciation de la collusion du pouvoir et des élites savantes, également virulente. C'est au contraire un champ à conquérir. Autour de Darwin se joue le rapport même à la science.

L'idée générale (cette petite somme des lois de l'homme et de la Terre) dont parle Reclus en 1894, il la formule dans L'Évolution, la révolution et l'idéal anarchique (1898), où il explique comment se fait l'humanité. Le livre de 1898 a lui-même mûri, depuis une conférence à Genève en 1880, Évolution et révolution, devenue petite brochure en 1880 (25 pages), devenue grosse brochure en 1891 (61 pages), puis livre entier.

Dès le départ, il s'agit d'une parole de science humaine et sociale : voici, argumente-t-il, la révolution enfin comprise avec des moyens scientifiques (en rivalité avec Marx). Les trois états du texte, de la brochure au volume $(1880,1891,1898)$, font apparaître un point fixe: la réfutation du "darwinisme social», c'est-à-dire le refus de toute instrumentalisation de Darwin qui viserait à justifier la violence des rapports sociaux. Reclus discerne deux systèmes qui entendent justifier l'exploitation de l'homme par l'homme, la religion d'une part, d'autre part la théorie du « droit du plus fort », qui «a du moins le mérite de ne pas reposer sur un mensonge » : « La théorie de Darwin vient de faire son entrée dans la science et l'on croit pouvoir s'en servir contre nous. $»^{15}$ (1880)

18 Sa réponse consiste à accepter le constat (la force règne), en ajoutant : bientôt nous serons les plus forts, la révolution sera l'avènement de la nouvelle force. Si Darwin sacre le plus fort, c'est nous que Darwin sacre. "Car si le capital garde la force [...] L'humanité aura cessé de vivre $»^{16}:$ or l'humanité lutte pour vivre, elle ne peut qu'être la plus forte, donc le capital mourra.

En 1891 la même démonstration (avec plus d'emphase et de développements) présente une variante menue mais significative : "La théorie dite de Darwin vient de faire son entrée dans la science et l'on croit pouvoir s'en servir contre nous. ${ }^{17}$ Dans le livre de 1898, la distance introduite entre Darwin et le « darwinisme social » s'accroît encore : "On invoque contre les revendications sociales le droit du plus fort, et même le nom respecté de Darwin a servi, bien contre son gré, à plaider la cause de l'injustice et de la violence. $»^{18}$

20 En même temps se développe l'ambition de formuler une loi sociale, en somme d'être le Darwin du monde social et de formuler « la vie profonde de l'Humanité ${ }^{19}$, en réflexion sur la force et la violence. Les temps ne sont plus à la force physique, y compris en révolution, écrit Reclus. Plus les révolutionnaires développent la conscience de la Révolution, plus cette dernière se confond avec l'évolution: «Finalement, toute opposition devra céder et même céder sans lutte [...] C'est ainsi que fonctionne la vie dans un organisme sain, celui d'un homme ou celui d'un monde $»^{20}$ (conclusion de 1891 et de 1898).

21 Les plus forts étant ceux qui s'entraident, l'entité destinée à être la plus forte est l'humanité enfin une. Tout le cheminement de Reclus, depuis la conférence de 1880, consiste à formuler une loi de la force qui ne soit pas une loi de la violence. D'où le balancement d'évolution et de révolution, au profit du mouvement présenté comme le plus naturel (la vie d'un organisme sain), et la mise en valeur de la conscience révolutionnaire, définie comme la connaissance des « lois » qui régissent l'humanité en devenir. 

prennent position contre le "darwinisme social », c'est-à-dire contre tous ceux qui défendent l'état de fait au nom d'une loi naturelle du plus fort. Dans cette controverse, l'objectif est de récupérer Darwin, de se positionner comme ses meilleurs lecteurs et interprètes.

fétiches : « lutte », « forts », « vie », « évolution ». En rejetant une interprétation et une instrumentalisation (elles-mêmes stéréotypées), le discours, quoiqu'il soit inverse ou précisément parce qu'il l'est, passe toujours par les mêmes mots (comme les jouteurs du Cromwell de Hugo qui se battent à coups de citations bibliques).

24

«Lutte » et « vie » sont ainsi ré-agencées par Jean Grave (dans la brochure de $1889: L a$ société au lendemain de la Révolution). À l'être égoïste et limité, il substitue, en citant Büchner, le grand être collectif, la lutte pour la vie de l'humanité, «pour la vie en général », la lutte contre la nature pour le bien-être de tous ${ }^{21}$.

L'« évolution» est travaillée par Émile Gautier (Le Darwinisme social, brochure anarchiste de 1880) et Jean Richepin (qui résume la brochure de Gauthier avec beaucoup de sympathie, en 1882). C'est une joute contre Haeckel (qui lui-même répondait en 1879 à Virchow, lequel avait affirmé que le système de Darwin donnait des arguments au socialisme). Gautier et Richepin affirment, contre le darwiniste Haeckel (et non contre Darwin), un «darwinisme social » retourné, qu'ils définissent comme socialiste par nature. Il s'agit donc de poser Darwin (le vrai) comme un enjeu de la disputation théorique. "Oui [...] l'homme primitif était soumis à cette loi et à ces conséquences alors fatales. Mais non plus l'homme d'aujourd'hui. $»^{22}$ L'homme évolue (dixit Darwin), il a accédé à la conscience, source de la société ; la conscience dresse l'homme contre la nature ; conclusion : "la société a d'abord été et sera de plus en plus une réaction contre la loi de Darwin $»^{23}$. - Autrement dit: contre la loi de Darwin et cela en vertu de Darwin. «La loi de Darwin» doit rencontrer des «conditions nouvelles » : la loi de la lutte mène l'homme hors de la lutte contre lui-même, vers l'entraide humaine (« les vrais forts de par leur nombre » et de par « leurs énergies latentes»), tournée en lutte contre la nature... Autour de cette même idée, nombreuses sont les interventions de Kropotkine : les «faibles» qui s'aident sont plus forts que les loups solitaires, la « vie » (le gros lot de la lutte), est donc à eux ${ }^{24}$, au terme d'une évolution qui doit faire de celui qui est ou a été le "plus fort» celui qui sera le plus faible. C'est à qui actionnera la balançoire, et remontera en faisant redescendre l'autre.

Il faut prendre toute la mesure de ce formatage du raisonnement : la réflexion joue à l'intérieur d'un cadre fixe, avec la contrainte de certains mots, et avec la contrainte première de prononcer «scientifiquement» (en s'élevant au niveau de la loi, de la généralité) sur l'homme, et l'humanité. « La vie » est en jeu : à qui reviendra-t-elle ? La grande formule de «la lutte pour l'existence », lancée par Darwin, débouche sur une lutte rhétorique autour de Darwin : c'est une lice où personne, manifestement, ne veut être dit le faible (ou alors et seulement le faible qui sera le fort). Ce n'est pas tant le détail d'une recherche scientifique qui agite le débat, mais la question viscérale, brutalement efficace, de prononcer la mort ou la vie de telle catégorie, de telle entité, de telle identité. La science devenue lutte de mots et pour les mots n'est pas forcément dévitalisée : au contraire. Sans doute elle entretient des clichés et des échanges de clichés, ils servent cependant une lutte à vie - à mort.

Arts et Savoirs, 12 | 2019 


\section{Topoi michéliens}

L'implication de Louise Michel dans ce débat et combat ne peut surprendre. Darwin se trouve mouliné dans un système de pensée et d'imaginaire drastiquement tendu par

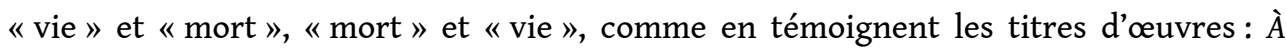
travers la vie, intitulant le premier recueil de poèmes en 1888, À travers la mort intitulant le second recueil poétique, qu'elle n'a jamais publié ; À travers la mort intitulant d'après les manuscrits de $1891^{25}$ le « second volume » des Mémoires (paru en feuilleton en 1890), et À travers la vie intitulant rétrospectivement, dans les mêmes plans, le premier volume des Mémoires (paru en librairie en 1886). Ces « à travers » configurent le sas du grand retournement : celui du monde ancien (qui va jusqu'au présent inclusivement, pour Louise Michel) et du monde nouveau, où l'homme sera un autre être humain, ayant fait sa nouvelle (r)évolution (nouvel animal et nouvelle société). Grand retournement aussi des « chassés » et des « chasseurs » (autre nom des faibles et des forts dans la lutte pour la vie, traduite en images de chasse à l'homme), selon la métaphore que le roman de $L a$ Chasse aux loups porte à son climax en 1891, en imaginant la revanche des morts de 1871 et le retour victorieux de la Commune.

L'extrême intérêt de Louise Michel pour la préhistoire, le moment le plus chaud de la «lutte pour la vie», marque aussi bien la production fictionnelle, autobiographique, propagandiste, encyclopédique. C'est là qu'elle trouve preuve d'évolution, non seulement organique (depuis le protoplasme) mais sociale : la lutte pour la vie a produit la société c'est-à-dire l'entraide, qui produira la révolte (la volte-face) des chassés contre les chasseurs. Ainsi dans Prise de possession (brochure publiée en 1890), elle montre les deux extrémités du cercle (l'homme préhistorique et l'homme contemporain) se rejoignant pour fermer un «cycle» et déboucher sur un nouveau cycle, à la fois en rupture et en évolution ${ }^{26}$.

29 La conversion de la lutte pour l'existence en lutte contre la nature (par les hommes réunis et s'entraidant), répétée à maintes pages, mise en scène dans les romans, avoue son implicite dans tel fragment encyclopédique: à l'époque tertiaire, écrit-elle: " chaque jour, dit Darwin, assurait la lutte pour vivre ». Elle ajoute : "Que la science et le travail fassent l'existence facile et qu'on lutte bientôt contre les forces de la nature pour la science et la vérité [biffure du manuscrit] pour le bonheur du genre humain. »

C'est sur la vie (à venir) du grand être «humanité » que débouche la lutte pour la vie originelle et présente :

L'humanité

Humanité ! mot encore vide de sens ou plutôt ne représentant que l'âpre lutte pour l'existence mais qui aura son accomplissement.

Cette lutte, partout sanglante, commence avec la vie, elle passe en héritage des pères aux enfants d'autant plus implacablement que les jours s'écoulent.

L'Humanité, c'est encore le troupeau presque tel que le dépeint Horace, le troupeau sortant aux premiers jours hideux et muets en rampant sur la terre nouvelle.

N'est ce pas, c'est toujours la force, comme au commencement. ${ }^{27}$

\section{Travaux pratiques. Ravachol le brachyne}

31 Chasse à l'homme et anthropophagie: Louise Michel, pour dire l'état social, recourt systématiquement au clivage des chasseurs et des chassés, des mangeurs et des mangés, 
et en cherche le dépassement dans l'évolution des espèces. La métaphore animale, prégnante, a ses rails : s'il s'agit de tuer le loup, figure récurrente du prédateur (et marquée du double sceau de Hobbes et de Darwin), il s'agit plus encore de penser sa transformation. Ainsi dans les romans de 1888-1889, le personnage de loup, nommé Wolff, apparaît comme progéniture d'un "grand ancêtre », un «fauve ancestral » et bestial, mais père de petits Wolff très humanisés : preuve de leur délupisation (pour ainsi dire), le roman les nomme des "loulous " (c'est-à-dire des chiens, en argot). Le père prédateur (et brillant scientifique) est déchiré lui-même entre le passé monstrueux et l'appel du monde nouveau. Par ailleurs un autre Wolff surgit dans le roman suivant: et celui qui porte ce nom est, sinon un "loulou», du moins un révolutionnaire et anarchiste ${ }^{28}$, passé du bon côté de la lutte (Le Claque-dents).

Il s'agit aussi, inversement, de penser l'évolution des mangés, des moutons, afin que leur nature ne soit plus de se laisser croquer. Les « troupeaux » qui se laissent tondre et saigner (par la République, par la démocratie élective...) sont aussi responsables que les loups (aussi peu, ou tout autant) : «Le nombre immense de profils de moutons chez tous les peuples explique la facilité avec laquelle peuvent s'accomplir les égorgements. $»^{29}$ Bref, il faut revoir les espèces sociales : si le "loup " a vocation à s'adoucir en loulou pour le bien de l'humanité ou à se convertir lutteur de la révolution, le «mouton» doit inversement gagner en combativité. L'actualité fournit à Louise Michel l'indice de l'évolution. Quand, en janvier 1886, les mineurs de Decazeville défenestrent le sous-directeur Watrin, elle déclare que «le moment est venu pour l'humanité » de faire la "chasse aux loups ». Eugène Pottier écrit au même moment "La revanche des moutons $»^{30}$, qui décrit une révolution autant qu'une métamorphose : des crocs poussent aux moutons, « Gare là-dessous! », ils " vont manger les loups! »

L'article que Louise Michel donne à la presse socialiste en août 1892, "À propos des explosions », pour expliquer l'attentat de Ravachol (qui vient d'être guillotiné), livre sa pensée de manière paradigmatique, et mieux encore, éclaire sa pratique de la référence scientifique. Rhétoriquement tout respire l'allusion à la vulgate darwinienne telle qu'elle est élaborée par le courant anarchiste, dès le mot de "lutte ", dès l'incise sur «l'humanité ne voulant pas périr »:

Il est naturel de jeter un coup d'œil sur les nouvelles formes que revêt la lutte sociale.

[...] La lutte entre le monde qui s'écroule et celui qui cherche à naître devait changer de forme. - Les écrasements de multitudes ont eu pour conséquence (l'humanité ne voulant pas périr) la lutte seul à seul, les armes ne peuvent donc être les mêmes.

Cette conséquence est instinctive. Les hommes ne s'adaptent-ils pas, pour vivre, aux lieux qu'ils habitent, n'ont-ils pas subi, en changeant leur mode d'existence, les changements d'époque, se défendant contre les périls qui les menacent par les moyens les plus propres à les conjurer. ${ }^{31}$

Tel est le préambule théorique. Les multitudes ne sont pas opérantes : le pouvoir les écrase. L'humanité continue cependant de vouloir vivre et de se battre pour son existence de grand être « Humanité ». Ravachol, individu ("seul à seul»), incarne à la fois cette lutte de l'humanité pour la vie, et la capacité (ou la nécessité) de s'adapter, organiquement. La « lutte sociale » change de «forme », et lui de même. Par la vertu de l'image il se métamorphose, il devient un insecte doué de certaines capacités singulières :

Un pauvre petit coléoptère, la brachine $[s i c]$ se défend des gros insectes en les effrayant au moyen de fusées qui se développent sous ses ailes; il est évident que la 
nature l'a poussée à employer ce moyen, serait-elle moins puissante pour empêcher la destruction de l'humanité?

[...] L'extermination a toujours été conjurée par les êtres capables de s'adapter à un milieu plus élevé.

Justifier Ravachol consiste à voir en lui, scientifiquement et de par la loi de Darwin, la métamorphose de l'animal humain. Comme le brachyne, "pauvre petit coléoptère » poussé par la nature à se défendre, à adapter son corps à la lutte, il a secrété sa fusée. On comprend l'humour du titre («À propos des explosions»), mais aussi sa portée : Louise Michel naturalise l'attentat à l'explosif.

Revenons sur le brachyne. Cet insecte avait eu les honneurs de l'Encyclopédie nouvelle de Reynaud et Leroux en 1840, qui distingue le brachinus crepitans, le brachinus explodens et le brachinus sclopeta :

[...] genre de coléoptères, l'un des plus remarquables qui existent dans la classe entière des insectes, par la faculté que possèdent les espèces qui le composent, d'émettre, avec explosion, par l'anus, une matière acide et vaporisable lorsqu'on les saisit ou les inquiète de quelque manière que ce soit [...] Les trois espèces [sont]: Brachine pétard (B. crepitans), brachine à explosions (B. explodens), brachine pistolet (B. sclopeta). ${ }^{32}$

L'insecte est du reste suffisamment fameux pour figurer dans un manuel à destination des enfants, en 1882, et pour y être dessiné en pleine action ${ }^{33}$. Le manuel est dû à JeanHenri Fabre, naturaliste et entomologiste, auteur d'un grand nombre d'ouvrages pour l'enseignement élémentaire à partir des années 1860 . On trouvera l'illustration en fin d'article.

L'article «À propos des explosions » fait comprendre comment la science s'intègre à un arsenal intellectuel, lui-même au service d'une lutte et d'un espoir placé dans le futur, gagé sur la transformation de l'animal humain. Le jet d'explosif est un processus « naturel » de l'évolution, comme le « coup d'œil » de Louise Michel sur la situation est «naturel» (selon ses mots). Le brachinus, qu'il soit crepitans, explodens ou pistolet, réoriente, darwinise et corse la métaphore romantique usée de la chrysalide, du reste omniprésente chez Louise Michel. Il métamorphose l'image de la métamorphose.

En lançant sa bombe, Ravachol-brachyne conchie tout simplement la justice et l'état social. Outre Darwin, un autre auteur se devine en filigrane de l'article de Louise Michel, le Hugo des Misérables et plus précisément celui du chapitre sur Cambronne ${ }^{34}$. À Waterloo, devant l'écrasement adverse, Cambronne « fait plus que cracher », il «noie dans deux syllabes la coalition européenne »: «sous l'accablement du nombre, de la force et de la matière, [il] trouve à l'âme une expression, l'excrément ». Cambronne lâchant son mot à l'ennemi surpuissant (dans la version hugolienne et non pas historique des faits), cela entrait dans un système du sublime, par la conjonction des extrêmes : le héros visité par le souffle se défendait, sauvait la France vaincue, par le Verbe « faisant explosion ».

Dans l'opposition des mangeurs et des mangés, l'agneau ou l'oisillon excitent la compassion tendre. Les traits traditionnellement attachés aux moutons n'entraînent en revanche pas d'affect empathique ni d'emballement esthétique, à la différence du loup, $\mathrm{du}$ fauve, de l'habitant sauvage des grands bois, beau de monstruosité, sublime d'horreur, dont les appétits furieux donnent des ailes à l'écriture feuilletonesque. Entre ces moutons, dont la passivité pose problème, et la chrysalide, qui constitue une image usée jusqu'à la corde, la trouvaille du brachyne restaure toute la saveur, toute la joie et la sublimité romantiques du «faible » qui se redresse, de l'écrasé qui tient tête et qui, 
désarmé, perdu, invente ses armes en les crachant, et en faisant plus que cracher, à la figure de l'ennemi. "L'avenir est dans les faibles", disait Michelet dans son cours de décembre 1847. En 1859, dans la Légende des siècles, Hugo dressait son Satyre ${ }^{35}$ contre les Olympiens. Le Ravachol-brachyne de Louise Michel appartient à cette grande famille des faibles qui sont des ressorts de force, des écrasés dont l'écrasement même réinvente l'explosion. Il est à mi-chemin du Cambronne de Hugo et de l'insecte de Michelet, cet insecte champion des métamorphoses tirées de son propre fonds, d'une sorte de création organique et morale de soi-même ${ }^{36}$.

41 L'article sur Ravachol est allègrement conscient de la provocation qu'il contient. Ce n'est évidemment pas une dissertation scientifique protocolaire, qu'il serait absurde d'attendre dans un contexte qui cherche à réinventer le savoir, à le gorger d'affect, d'imaginaire et d'action. Cependant la référence darwinienne n'est ni insignifiante, ni indifférente, ni inopérante : elle est au centre de l'argumentaire, constituant l'ossature du texte, de la pensée et de l'imaginaire. Elle est d'autant plus sérieuse qu'elle est ouvertement sardonique, et inversement. Comme Ravachol-Brachyne se sert de la science (c'est-à-dire de la chimie et des explosifs) pour lancer des "fusées ", de même Louise Michel se sert de la science (c'est-à-dire de la référence darwinienne) pour justifier Ravachol : elle aussi s'adapte et adapte les ressources. La lutte pour la vie fait feu de tout bois. C'est dans cette logique paradoxale, circulaire, sciemment déformante, mais vivante, que se trouve pris, amalgamé et emporté le Darwin de la «lutte pour la vie ».

Tel est le Darwin fin de siècle, anarchiste et nourri de romantisme, qu'élabore Louise Michel. Elle pleure sur les troupeaux, mais ne s'identifie pas à eux; sans doute elle entend leur prêcher la révolte, attiser toutes les déflagrations, mais c'est l'acte anarchiste pour en découdre « seul à seul » qui stimule la production d'images, illustre et sublime l'évolution révolutionnaire. Dans cette fable moderne de David et de Goliath, - ou de Darwin et de Goliath - le geste de Ravachol vaut comme une démonstration de l'évolution. Le terroriste guillotiné, en tant qu'anarchistus explodens, porte la légende héroïco-scientifique de la lutte pour la vie. «Et puis, voyez-vous, il n'y a pas à dire : nous sommes dans la grande lutte entre le vieux monde et le nouveau. La lutte a commencé, elle est implacable; il faut qu'elle aille jusqu'au bout ${ }^{37}$, déclare Louise Michel dans une interview de la fin 1893, posant entre la Géographie universelle d'élisée Reclus, posée en évidence sur la table et, sur le piano, une partition de «La Tempête ». « Non, il n'y a de vrai que la lutte! la lutte où nous savons que nous resterons! Et c'est là le beau ! ». Telle est, répète-t-elle, la « légende moderne » fondée par Ravachol ${ }^{38}$. 
Figure

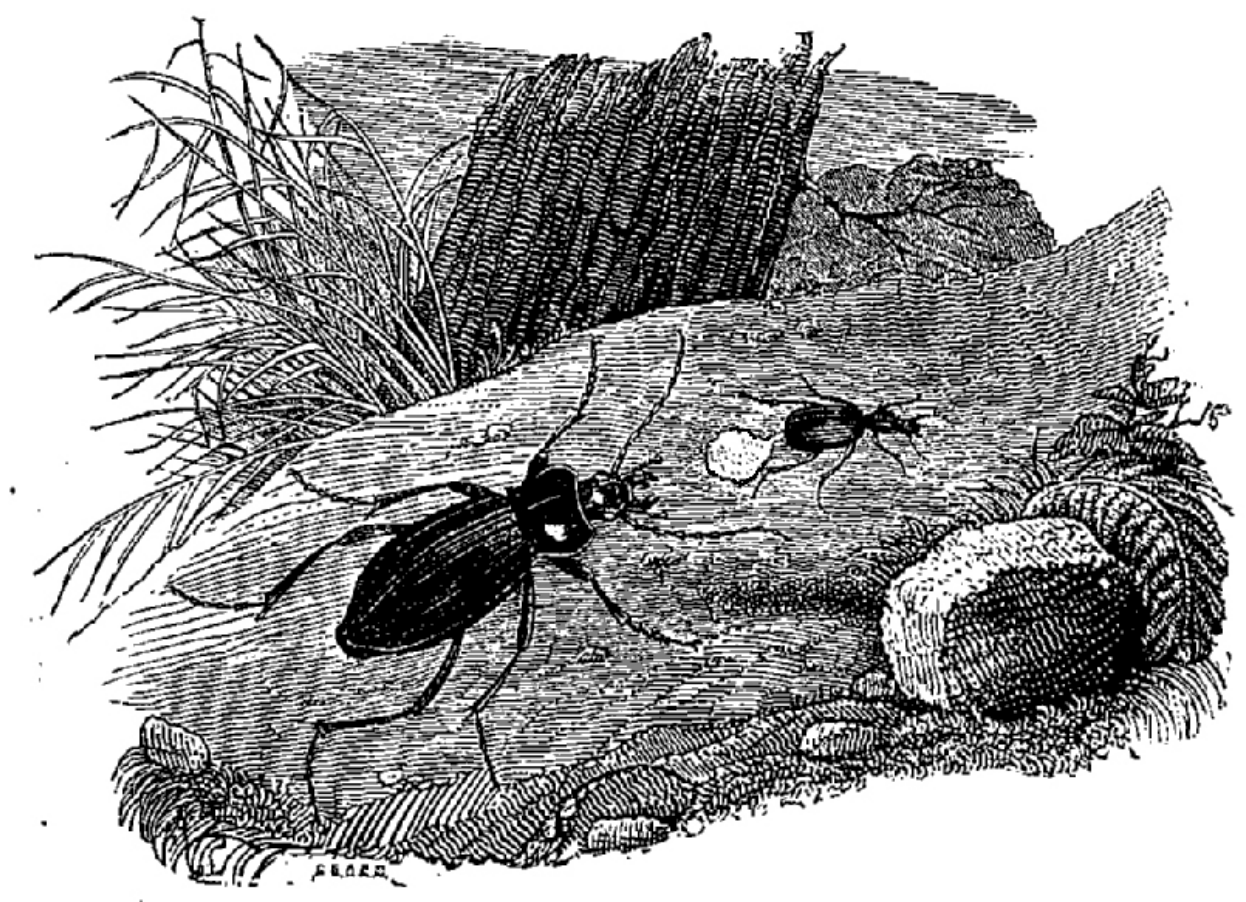

Fig. 40. - Brachine en défense contre un Carabe.

Jean-Henri Fabre, La Science élémentaire, lectures pour toutes les écoles, op. cit.

Une version de cet article a été précédemment publiée dans Biological Time, Historical Time. Transfers and Transformations in 19th Century Literature, Niklas Bender et Gisèle Séginger (dir.), Brill | Rodopi, 2018, « Faux-Titre », p. 389-406, https://brill.com/view/title/39098.

\section{NOTES}

1. Un essai consacré au corpus encyclopédique de Louise Michel et un recueil de textes paraîtront prochainement (Claude Rétat, Paris, Classiques Garnier).

2. Voir Marc Angenot, 1889, Un état du discours social, Longueuil, Éditions du Préambule, 1989 (en particulier le chapitre XL: "Migrations d'un idéologème: "La lutte pour la vie" ») ; Jean-Marc Bernardini, Le Darwinisme social en France (1859-1918), Paris, CNRS Éditions, 1997.

3. Louise Lyle, «Charles Darwin dans Le Jardin des supplices ", Cahiers Octave Mirbeau, ${ }^{\circ} 14$, mars 2007, p. 47-64.

4. D'après Le Socialiste, 26 septembre 1885. Le livre de Darwin, The Descent of Man, and Selection in Relation to Sex (Londres, John Murray, 1871, 2 vol.), a été traduit en français en 1872 ( $\mathrm{La}$ Descendance de l'homme et la sélection sexuelle).

5. Ms de Moscou, RGASPI, 233/1/5. Ce texte réfère à Darwin, The Descent of Man, op. cit., vol. I, p. 53-54.

6. Louise Michel, Trois romans, Les Microbes humains, Le Monde nouveau, Le Claque-dents, éd. Claude Rétat et Stéphane Zékian, Lyon, PUL, 2013, p. 202. 
7. Louise Michel, À travers la mort, Mémoires inédits, 1886-1890, éd. critique Claude Rétat, Paris, La Découverte, 2015, p. 270.

8. Louise Michel, Lectures encyclopédiques par cycles attractifs [...], première livraison, Paris, Librairie d'éducation laïque, 1888, p. 12.

9. Selon le titre de l'ouvrage de Darwin: On the Origin of Species by Means of Natural Selection, or the Preservation of Favoured Races in the Struggle for Life (Londres, John Murray, 1859).

10. Voir Claude Rétat, Art vaincra! Louise Michel, l'artiste en révolution et le dégoût du politique, SaintPourçain, Bleu autour, 2019 (en particulier p. 20, 60-66, 130). L'imaginaire de la transformation nourrit chez Louise Michel à la fois une cosmogonie et le rejet du monde politique : à l'animal humain qui se métamorphose (et autour duquel le monde se recrée), elle oppose le caméléon, l'opportuniste, le spécialiste de la reproduction du vieux monde.

11. Ibid., p. 16.

12. Louise Michel, Notions encyclopédiques par ordre attractif, P. Buchillot printer, s.d. [ca 1894], p. 1.

13. « Dernier mot », par Élisée Reclus, dans L'Intransigeant, 3 janvier 1894.

14. Ibid.

15. Élisée Reclus, Évolution et révolution, Genève, Imprimerie Jurassienne, 1880, p. 22-23.

16. Ibid., p. 25.

17. Élisée Reclus, Évolution et révolution, Paris, Au bureau de La Révolte, 1891, p. 35. Je souligne.

18. Élisée Reclus, L'Évolution, la Révolution et l'idéal anarchique, Paris, Stock, 1898, p. 203. Je souligne.

19. Ibid., p. 193.

20. Ibid., p. 291-292 (et brochure de 1891, op. cit., p. 61).

21. Jehan Le Vagre [Jean Grave], La Société au lendemain de la révolution, Paris, Au bureau de La Révolte, 1889, p. 124-125.

22. « Le darwinisme social », par Jean Richepin, Gil Blas du 25 octobre 1882.

23. Ibid. En italique dans le texte.

24. Kropotkine, «L'appui mutuel chez les sauvages », Supplément littéraire de La Révolte, ${ }^{\text {os }} 21$ et suivants [1891].

25. Voir Louise Michel, À travers la mort, Mémoires inédits..., op. cit., annexe 2, p. 303.

26. Louise Michel, Prise de possession, éd. Claude Rétat, Paris, L'Herne, 2017, p. 34-35.

27. Ms de Moscou, RGASPI, 233/1/4.

28. Voir Le Monde nouveau (Trois romans, op. cit.) où Wolff meurt poursuivi par la foule, et Le Claque-dents (ibid.) où Wolff est le nom d'un révolutionnaire.

29. IISH (International Institute of Social History, Amsterdam), Louise Michel papers, ms 675.

30. Le Cri du peuple, 9 et 10 février 1886.

31. La Question sociale, 15 août 1892. Texte reproduit en annexe de La Chasse aux loups, éd. critique Claude Rétat, Paris, Classiques Garnier, 2018 (2015), p. 327 ; voir ibid. la présentation du roman, p. 37 et suiv.

32. Encyclopédie nouvelle, Pierre Leroux et Jean Reynaud (dir.), t. III, Paris, Gosselin, 1840, p. 54.

33. Jean-Henri Fabre, La Science élémentaire, lectures pour toutes les écoles, Paris, Ch. Delagrave et $\mathrm{C}^{\mathrm{ie}}$, 1882, p. 174

34. Victor Hugo, Les Misérables, $\mathrm{II}^{\mathrm{e}}$ partie, livre $\mathrm{I}^{\mathrm{er}}$, chap. XV (๕euvres complètes, éd. Jean Massin, Paris, Le club français du livre, vol. XI, 1969, p. 281-282).

35. $3^{\mathrm{e}}$ leçon du Cours au Collège de France : «L'avenir est dans les faibles » (30 décembre 1847), dans Jules Michelet, OC, Bible de l'Humanité. Une année au College de France, E. Flammarion, s. d. Victor Hugo, Légende des siècles, Première série.

36. Jules Michelet a publié L'Insecte en 1858. Voir en particulier le livre $\mathrm{I}^{\mathrm{er}}$, chap. VI: « Métamorphose. La momie, nymphe ou chrysalide ».

37. « Louise Michel. L'opinion de la Vierge rouge sur l'anarchie », Le Matin, 19 décembre 1893.

38. Ibid. 


\section{RÉSUMÉS}

La référence darwinienne, topique à la fin $\mathrm{du}_{\mathrm{XIX}}^{\mathrm{e}}$ siècle, est au centre d'une joute rhétorique à qui sera (ou dira qu'il est) du bon côté de l'histoire et du bon côté dans la lutte pour la vie. Louise Michel articule les topoi de l'évolution, et avec eux les topoi de l'évolution-révolution, à un imaginaire et à une pratique (de pensée, d'écriture, d'engagement militant). Son article de 1892, "À propos des explosions", sur l'attentat de Ravachol, fait apparaître de manière paradigmatique, sardonique et originale, son usage de la référence scientifique. Ou comment Ravachol, de par la science et la loi de Darwin, se métamorphose en une nouvelle sorte de Cambronne et fonde la « légende moderne ».

The reference to Darwin, topic at the end of the 19th century, is at the heart of a verbal sparring match about who will be (or will claim to be), in matters of struggle for life, on the right side of history. Louise Michel articulates the topoi of evolution, and alongside the topoi of evolutionrevolution, with an imagination and a practice (of thought, of writing, of militant engagement). Her 1892 article "À propos des explosions" ("On explosions", treating the assassination attempt of Ravachol) shows in a paradigmatic - but nonetheless sardonic and original-way her use and practice of scientific reference.

\section{INDEX}

Mots-clés : Michel (Louise), Darwin, science, lutte pour la vie, évolution, révolution

Keywords : Michel (Louise), Darwin, science, struggle for life, évolution, revolution

\section{AUTEUR \\ CLAUDE RÉTAT}

CNRS, UMR 8599-CELLF 